\title{
Recurrent Urinary Tract Infection in an Elderly Man
}

\author{
Lung-Yi Mak ${ }^{\mathrm{a}, \mathrm{c}}$, Tuen-Ching Chan ${ }^{\mathrm{b}}$, Hon-Wai Chan ${ }^{\mathrm{b}}$, \\ Ivan Fan Ngai Hung ${ }^{a}$
}

\begin{abstract}
An elderly man with newly diagnosed dilated cardiomyopathy and benign prostatic hypertrophy presented with classical symptoms of complicated urinary tract infection (UTI) in a recurrent manner. A simple investigation, the plain abdominal X-ray, was overlooked and the diagnoses of staghorn stones and bladder stones were not made effectively. The outcome of UTI in men, which is often complicated, can be devastating. Surgical intervention can treat the root cause of recurrent UTI; however, in this gentleman with major medical comorbidity, limited options are available.
\end{abstract}

Keywords: Cardiomyopathy; Male; Nephrolithotomy; Pyelonephritis; Radiograph; Septicemia; Staghorn stone; Struvite stone; Urinary tract infection; Urolithiasis

\section{Introduction}

Urinary tract infection (UTI) in male is frequently associated with complicated clinical course; however, it is not uncommon in clinical practice. Many physicians are not aware of the essence of pursuing for the root cause of UTI in male, such as urolithiasis or bladder outlet obstruction; rather, male patients with UTI are often incorrectly managed in the same manner as female patients. Effort should be made, in particular at ordering as well as interpreting imaging, to look for urolithiasis as an essential step in defining etiology. A delay in diagnosing staghorn stone risks recurrent septic threats. This case report aims to illustrate the co-existing multiple risk factors for UTI in male, and to arouse the readers' awareness at the essence of abdominal radiograph for hinting the diagnosis of staghorn stones.

Manuscript accepted for publication August 12, 2016

aDepartment of Medicine, Queen Mary Hospital, University of Hong Kong, Hong Kong Special Administrative Region, China

bDepartment of Medicine and Geriatrics, Fung Yiu King Hospital, 9 Sandy Bay Road, Pokfulam, Hong Kong Special Administrative Region, China

${ }^{\mathrm{c} C}$ Corresponding Author: Lung-Yi Mak, Department of Medicine, Queen Mary Hospital, 102 Pokfulam Road, Pokfulam, Hong Kong Special Administrative Region, China. Email: loeymak@gmail.com

doi: http://dx.doi.org/10.14740/jmc2610w

\section{Case Report}

An 89-year-old gentleman with good past health was admitted for fever and right-sided abdominal pain. Initial physical examination was unremarkable apart from a tympanic temperature of $38.6^{\circ} \mathrm{C}$. Blood tests revealed leukocytosis (white cell count $20.2 \times 10^{9} / \mathrm{L}$, normal $\left.3.8-9.9 \times 10^{9} / \mathrm{L}\right)$ and elevated serum creatinine (creatinine $147 \mu \mathrm{mol} / \mathrm{L}$, normal 67 $-109 \mu \mathrm{mol} / \mathrm{L}$ ). Macroscopically, the urine sample appeared cloudy. Microscopic examination of urine showed presence of occult blood, pyuria (white cell count $>50 / \mu \mathrm{L}$ ), nitrites, leucocyte esterases, and of $\mathrm{pH}$ 6.0. Mid-stream urine culture grew Klebsiella pneumonia and Enterococcus species. An abdominal X-ray (AXR) was taken and regarded as "unremarkable". The chest X-ray incidentally detected cardiomegaly and echocardiogram revealed global hypokinesia with left ventricular ejection fraction of $20 \%$. The diagnosis was UTI and dilated cardiomyopathy and he was managed with oral augmentin $375 \mathrm{mg}$ three times daily for 1 week. The white cell count normalized and he was discharged home after defervescence of fever.

However, in 2 months' time, he presented with recurrence of right-sided abdominal pain, fever and septic shock. On admission, the blood pressure was 90/48; pulse rate was 91 beats per minute; tympanic temperature was $40^{\circ} \mathrm{C}$. There was marked leukocytosis (white cell count $25.1 \times 10^{9} / \mathrm{L}$ ), thrombocytopenia (platelet count $88 \times 10^{9} / \mathrm{L}$, normal $154-371 \times 10^{9} / \mathrm{L}$ ), coagulopathy (prothrombin time $14.0 \mathrm{~s}$, activated thromboplastin time $54.1 \mathrm{~s}$; normal $11.3-13.5 \mathrm{~s}$ and $25.9-33.7 \mathrm{~s}$, respectively) and further impairment of renal function (creatinine: $300 \mu \mathrm{mol} / \mathrm{L}$ ). Blood and urine culture yielded Klebsiella pneumoniae. Ultrasound examination showed benign prostatic hypertrophy $(\mathrm{BPH})$ and an echogenic shadow at right renal pelvis. Upon retrospective review of the initial AXR taken 2 months ago, there was a staghorn shaped radio-opaque shadow over the right renal pelvis and another oval shaped shadow over the suprapubic fossa (Fig. 1). Computed tomography (CT) of the abdomen confirmed a right-sided staghorn stone causing blunting of right renal calyces and diminished size of right kidney (Fig. 2). Another stone was seen at right side of urinary bladder obstructing the right vesico-ureteric junction (Fig. 3). There was no hydronephrosis. Bilateral symmetrical perinephric stranding was demonstrated. He was treated with intravenous meropenem $500 \mathrm{mg}$ every $12 \mathrm{~h}$ and transferred to intensive care unit for inotropic support and close monitoring. His hemodynamic condition was stabilized and meropenem 


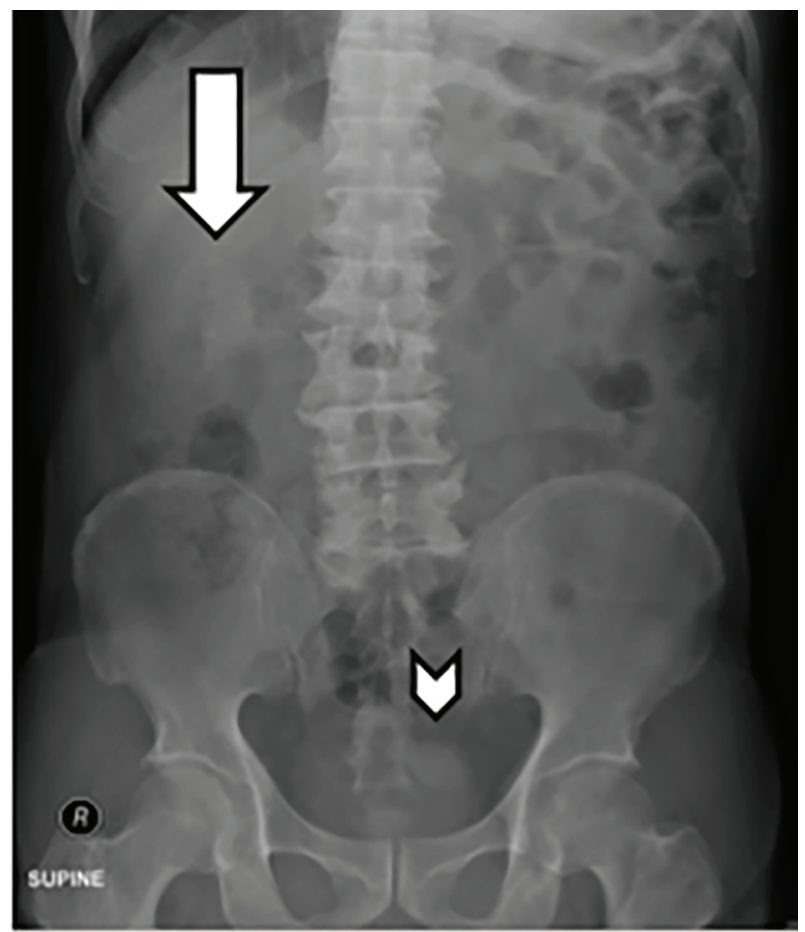

Figure 1. Plain abdominal X-ray. Arrow shows a staghorn stone in the right renal pelvis and chevron shows another stone in the urinary bladder.

was given for 2 weeks while all blood test parameters normalized. Urologist was consulted and suggested for long-term antibiotics for secondary prophylaxis, while surgical intervention was not indicated as the patient was of advanced age with underlying dilated cardiomyopathy. The patient remained asymptomatic and declined prophylactic antibiotics.

One year later, he developed another severe episode of UTI with Klebsiella bacteremia and septic shock, which failed to respond to broad-spectrum antibiotics and inotropic support, and he deteriorated rapidly and died.

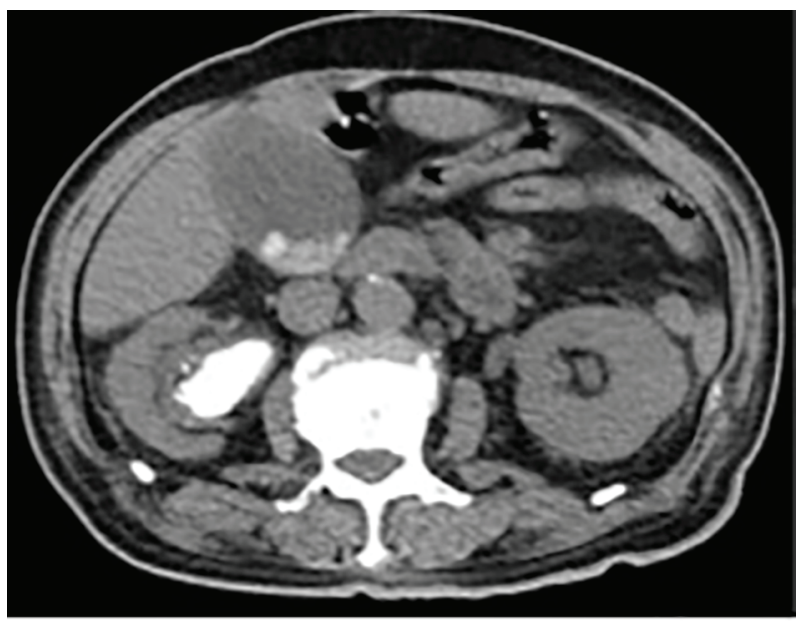

Figure 2. Computed tomography of abdomen showing right staghorn stone.

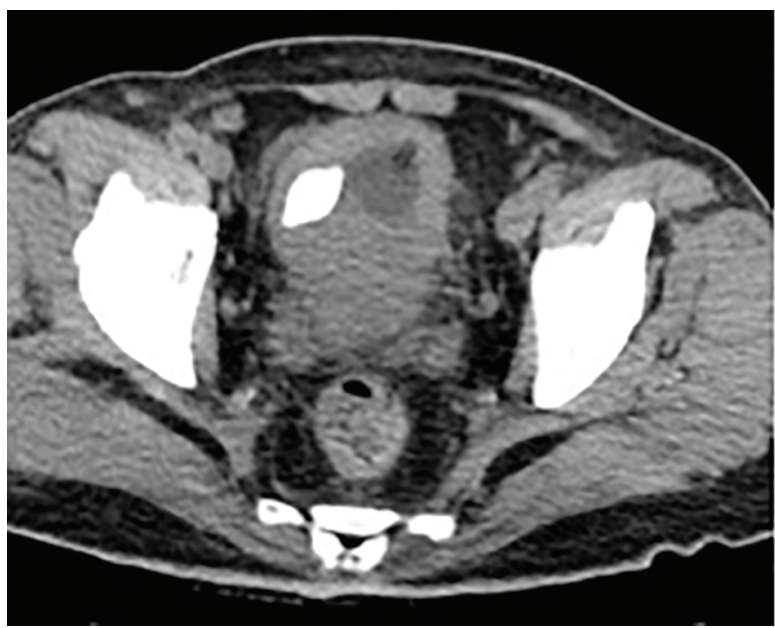

Figure 3. Computed tomography showing urinary bladder stone.

\section{Discussion}

Symptomatic UTI is much less common in men than in women [1]. It has been conventional to consider all UTIs in elderly men as complicated, which implies the presence of not only cystitis, but also pyelonephritis. The majority of UTIs in elderly men occur in association with urologic abnormalities, such as bladder outlet obstruction, urological instrumentation or presence of an infective nidus [2], therefore imaging is of utmost importance during evaluation of UTI in male. One of the important conditions predisposing an elderly man to UTI is struvite stones [3], which are composed of magnesium ammonium phosphate (struvite) and calcium carbonate-apatite. Such stones only form in the setting of an upper UTI with urea-splitting bacteria, such as Proteus or Klebsiella, and the urine will have an alkaline urine $(\mathrm{pH}>7)$, often with multiple magnesium ammonium phosphate crystals in the urine sediment. Struvite stones may grow rapidly over a period of weeks to months and, if not adequately treated, can develop into staghorn stones that fill the entire renal pelvis and calyces causing renal failure [4] and stone removal can improve renal function [5]. Therefore, most patients require adequate surgical treatment. The American Urological Association (AUA) recommends percutaneous nephrolithotomy (PCN) as the first-line approach, while shock wave lithotripsy can also be considered for smaller stones or in combination with PCN [3]. Medical therapy with chronic administration antibiotics rarely succeeds stone clearance, and should not be considered as sole treatment unless for patients who are too ill to tolerate, or refuse, stone removal via surgical means. Most renal calculi are readily shown on AXR and the reported sensitivity was 45-60\% [6]. It is the first investigation that can provide critical information which prompts further diagnostic evaluation of symptomatic UTI in elderly male, for example a CT scan. Physicians are taught to order AXR or kidney-ureter-bladder (KUB, another type of plain radiography), but the films are frequently not read or not interpreted, resulting in a delay in diagnosis and timely management.

In our patient, the primary diagnosis was right-sided stag- 
horn stone due to complicated Klebsiella UTI with pyelonephritis. The risk factor for UTI in this elderly man might be related to the underlying dilated cardiomyopathy and $\mathrm{BPH}$ which led to impaired glomerular filtration rate and urinary stasis. The staghorn stone, despite showing up on a plain AXR, was not diagnosed in the first episode of UTI, and he suffered from repeated episodes of UTI and further renal impairment. Nevertheless, diagnosing the stone 2 months earlier would not have altered the patient's outcome as the urologist decided for medical therapy alone after consideration of his age and comorbidity, since even though there was a treatable cause for his symptomatic UTI, he could not be benefited from surgical treatment.

\section{Conclusion}

This case demonstrated the fatality of an untreated struvite stone and the importance of detecting it early by imaging as simple as a plain AXR. Any AXRs taken should not be overlooked. Physicians should see UTI in elderly men seriously as they are often complicated and harbor significant predisposing causes that warrant prompt treatment.

\section{Grant Support}

No grant support for this case report.

\section{Author Contributions}

All authors have participated sufficiently in the intellectual content and analysis of data. Each author has reviewed the final version of the manuscript and approves it for publication.

\section{Conflicts of Interest}

None.

\section{References}

1. Vorland LH, Carlson K, Aalen O. An epidemiological survey of urinary tract infections among outpatients in Northern Norway. Scand J Infect Dis. 1985;17(3):277283.

2. Lipsky BA. Urinary tract infections in men. Epidemiology, pathophysiology, diagnosis, and treatment. Ann Intern Med. 1989;110(2):138-150.

3. Preminger GM, Assimos DG, Lingeman JE, Nakada SY, Pearle MS, Wolf JS, Jr. Chapter 1: AUA guideline on management of staghorn calculi: diagnosis and treatment recommendations. J Urol. 2005;173(6):1991-2000.

4. Jungers P, Joly D, Barbey F, Choukroun G, Daudon M. ESRD caused by nephrolithiasis: prevalence, mechanisms, and prevention. Am J Kidney Dis. 2004;44(5):799805 .

5. Gupta M, Bolton DM, Gupta PN, Stoller ML. Improved renal function following aggressive treatment of urolithiasis and concurrent mild to moderate renal insufficiency. J Urol. 1994;152(4):1086-1090.

6. Mutgi A, Williams JW, Nettleman M. Renal colic. Utility of the plain abdominal roentgenogram. Arch Intern Med. 1991;151(8):1589-1592. 